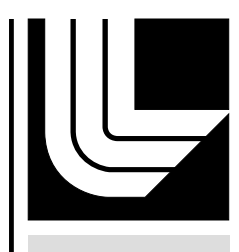

LAW RENCE LIVERMORE N A T IO N A L LABORATORY

\title{
UCRL-JRNL-236991
}

\section{A Quasicontinuum Study of Nanovoid Collapse under Uniaxial Loading in $\mathrm{Ta}$}

J. Marian, J. Knap, G. Campbell

December 4, 2007

Acta Materalia 
This document was prepared as an account of work sponsored by an agency of the United States government. Neither the United States government nor Lawrence Livermore National Security, LLC, nor any of their employees makes any warranty, expressed or implied, or assumes any legal liability or responsibility for the accuracy, completeness, or usefulness of any information, apparatus, product, or process disclosed, or represents that its use would not infringe privately owned rights. Reference herein to any specific commercial product, process, or service by trade name, trademark, manufacturer, or otherwise does not necessarily constitute or imply its endorsement, recommendation, or favoring by the United States government or Lawrence Livermore National Security, LLC. The views and opinions of authors expressed herein do not necessarily state or reflect those of the United States government or Lawrence Livermore National Security, LLC, and shall not be used for advertising or product endorsement purposes. 


\title{
A Quasicontinuum Study of Nanovoid Collapse under Uniaxial Loading in Ta
}

\author{
J. Marian, J. Knap and G. H. Campbell \\ Chemistry, Materials, Earth, and Life Sciences Directorate, Lawrence Livermore \\ National Laboratory, Livermore, CA 94551
}

\begin{abstract}
We analyze the mechanisms underlying the deformation of nanovoids in Ta single crystals subjected to cyclic uniaxial deformation using numerical simulations. Boundary and cell-size effects have been mitigated by means of the Quasicontinuum (QC) method. We have considered $\sim 1$ billion-atom systems containing 11.2$\mathrm{nm}$ voids. Two kinds of simulations have been performed, each characterized by a different boundary condition. First, we compress the material along the nominal [001] direction, resulting in a highly-symmetric configuration that results in high stresses. Second, we load the material along the high-index $\left[\begin{array}{lll}\overline{4} & 8 & 19\end{array}\right]$ direction to confine plasticity to a single slip system and break the symmetry. We find that the plastic response under these two conditions is strikingly different, the former governed by dislocation loop emission and dipole formation, while the latter is dominated by twinning. We calculate the irreversible plastic work budget derived from a loading-unloading cycle and identify the most relevant yield points. These calculations represent the first fully $3 \mathrm{D}$, fully non-local simulations of any bcc metal using QC.
\end{abstract}

Key words: Nanovoid collapse, Quasicontinumm method, shocks, strength of Ta PACS: 02.70.-c, 02.70.Uu, 61.72.Ss

\section{Introduction}

Understanding materials response to dynamic loading requires knowledge of the physical, mechanical and thermodynamic properties of materials over the appropriate pressure range. The different thermodynamic states and associated phase transitions are characterized by the equation of state (EOS), which may also give information about a wide range of mechanical states, including elastic behavior, plastic flow, and failure. The high-pressure EOS of materials is usually determined by shock compression experiments [1]. The data obtained 
from these experiments is then used to determine the pressure-volume-energy end states due to each shock wave experiment. The locus of several of these end states constitutes the so-called Hugoniot curve, which is a unique path on the EOS surface. The Hugoniot represents an adiabatic compression process by steady shock transitions to various final states, but it is highly irreversible, resulting in higher temperatures in the shocked state. In contrast, the so-called 'cold curve' is the isotherm at $T=0$, and represents the maximum theoretical compression that can be achieved by shock loading. If the compression process involves plasticity (hence no longer reversible), the cold curve then gives the total plastic work produced by deviatoric stresses available for irreversible heating. Therefore the cold curve is the reference from which temperature changes are computed. It is difficult to obtain isentropic states experimentally, and cold curves are usually estimated from theoretical Grüneisen models or other phenomenological approaches that include semiempirical information $[2,3]$.

Researchers are now endowed with more powerful computational approaches that can compute material properties with very few or no a priori assumptions. These methods, organized in hierarchical classifications (such as 'multiscale'), have been successfully tested for a number of pure systems, treating non-trivial aspects such as magnetism, phase transitions, etc. (for comprehensive reviews see, for example, Refs. [4,5]). However, modern engineering materials have increasingly complex microstructures. When subjected to extreme environments, such as high strain rates or shock-loading, understanding the response of the complex microstructures is key to modeling them. Specific features of these microstructures that may be relevent to their response include characteristics such as porosity that is found in any powder metallurgy material $[6,7]$, small voids or gas bubbles in irradiated materials [8], and the multiple phases that are found in composite materials [9]. The interaction among the phases, particularly during mechanical loading, can be complex and can give rise to unexpected responses. Efforts to properly model these systems are contingent on the calculation of accurate cold curves and the understanding of the related deformation processes. Therefore, in this work we use numerical simulations of uniaxially-loaded porous Ta as a model system to compute the associated cold curves and identify the unit mechanisms attendant to shock compression of these materials with complex microstructures.

The high experimental strain rates commonly reported for shock-loading experiments in metals - of the order of $10^{3}$ to $10^{6} \mathrm{~s}^{-1}$ - make atomistic techniques such as molecular dynamics (MD) ideally suited for the study of these processes [10-12]. However, the simulation of localized plastic phenomena in heterogeneous systems requires the use of very large systems and appropriate boundary conditions, which may result in system sizes difficult to access using direct MD. The Quasicontinuum method (QC) suggests itself as a viable alternative to fully atomistic methods, as it permits the treatment of long- 
ranged fields in a computationally efficient manner while resolving non-linear regions of the configuration space atomistically. The main shortcoming of QC is that it is a quasistatic technique and, as such, can only provide equilibrium configurations. However, quasistatic studies may also be helpful in studying the deformation pathways attendant to void collapse in Ta, as it has been the case in a number of other problems with similar geometric constrains to which QC has been successfully applied [13-15]. In addition, most atomistic studies have been carried out for $<5$-nm voids in fcc crystals under expansive conditions [14,16-19], with only a few focusing on void collapse [20-22]. Thus, despite the many benefits of MD, such as full spatial and temporal resolution, and the volume of work on small void compression, here we present results of $\sim 11$-nm void collapse in Ta obtained using QC. This represents three elements that are explored here for the first time using Quasicontinuum: relatively large (>10-nm) voids, bcc crystals, and compressive loading.

\section{Methodology}

\subsection{The Quasicontinuum method}

QC is a method for systematically coarse-graining lattice statics models [23,24]. The method starts with the complete atomistic system and reduces the configuration space of the crystal through judicious application of finite-elementbased kinematic constraints. In order to avoid full lattice sums, cluster summation rules are used. By virtue of these rules, only atoms in small clusters surrounding the representative atoms need be visited in computing the effective out-of-balance forces. Finally, the selection of representative atoms is performed adaptively based on a local strain measure and longest-edge tetrahedral bisection. The tolerances governing the adaption process are set such that full atomistic resolution is attained in the presence of dislocations. The QC method uses empirical potentials directly as the sole description of material behavior and contains fully atomistic lattice statics and continuum elasticity as special limits. As in conventional continuum mechanics, QC permits the direct simulation of systems controlled through the application of remote boundary conditions, a feature that is particularly useful in simulating dilute distributions of nanovoids. QC simultaneously provides atomistic resolution at defect cores without the stringent size limitations of direct atomistics. Details of the implementation of QC used in the present study and an analysis of the accuracy and convergence of the method may be found in [25]. For this work, an embedded-atom method (EAM) potential for Ta has been implemented [26] in QC. More details about the methodology and the potentials employed can be found in Section 4 . 


\subsection{Simulation details}

We study the response of Ta single crystal samples containing nano-sized voids to strong unidirectional shocks. We specifically consider two different loading orientations:

(i) Uniaxial compression along [001].

This involves loading a perfectly-spherical void along one of the principal axes of the cubic sample. Macroscopically, this deformation is characterized by the following (homogeneous) deformation gradient $\mathbf{F}$ :

$$
\mathbf{F}=\left(\begin{array}{ccc}
1 & 0 & 0 \\
0 & 1 & 0 \\
0 & 0 & \gamma
\end{array}\right)
$$

where $\gamma \geq 0$ is the prescribed macroscopic compression at each loading step. We define the accumulated axial strain as $\varepsilon_{z}=\frac{1}{2}\left[(1-\gamma)^{2 n}-1\right]$, where $n$ is the total number of loading steps. Here we have decreased $\gamma$ from 1.0 to 0.9 in decrements of half of a percentage point, i.e. $n \approx 20$.

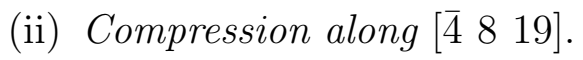

We have found a loading direction such that the difference in resolved shear stress (RSS) between the two most-highly-stressed slip systems is maximized. This is termed the maximum RSS margin orientation, and e.g. for slip on $[111](\overline{101})$ it lies close to the $\left[\begin{array}{lll}\overline{4} & 8 & 19\end{array}\right]$ slip direction. The primary [111](101) slip system then has a stress $\sim 13 \%$ higher than the next most highly activated system. In order to use the same crystallographic orientation as case (i), we have rotated a stress tensor with only one active diagonal component along the [ $\overline{4} 8$ 19] direction, $\sigma_{z}$, to the canonical frame of reference. In Appendix A we derive the corresponding deformation gradient, $\mathbf{F}^{\prime}$, in terms of the uniaxial stress $\sigma_{z}$. From eq. A.8, values of $0<\sigma_{z}<723.3$ GPa guarantee macroscopic compression, i.e. $J=\operatorname{det} \mathbf{F}^{\prime}=V / V_{0}<1$, where $J$ is the Jacobian, and $V$ and $V_{0}$ are, respectively, the current and reference total volumes (discounting the void volume). Here we have varied $\sigma_{z}$ from 0 to $11 \mathrm{GPa}$ in $1.0-\mathrm{GPa}$ increments.

Figure 1 shows these two crystallographic orientations represented in the stereographic triangle of the corresponding cartesian octant.

Another principal outcome of the calculations is the plastic work budget and degree of hysteresis associated with nanovoid collapse. In order to ascertain these aspects of the macroscopic behavior of the material we proceed to calculate the recovery curve resulting from the unloading and reverse-loading of 


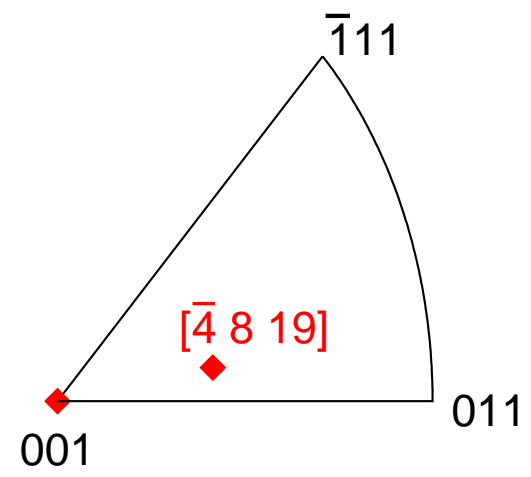

Fig. 1. Stereographic triangle in the $(x<0 ; y, z>0)$ octant containig the two axial loading orientations considered in this work (red diamonds).

the sample back to its initial macroscopic configuration. In case (i), we induce this unloading by restoring $\gamma$ to $100 \%$ following the attainment of its minimum prescribed value of $90 \%$. For case (ii), we simply decrease $\sigma_{z}$ from its maximum accumulated value of $11 \mathrm{GPa}$ to zero.

At each loading step, a new stable equilibrium configuration is obtained by means of the Polak-Ribiere variant of the conjugate gradient algorithm [27]. The computational mesh is then adapted so as to adequately resolve the fine features of the solution. The precise adaption indicator employed in the calculations reported here is as follows. Suppose that $\mathbf{F}$ is the local deformation gradient of a tetrahedron $K$ in the computational mesh. The departure of that local deformation $\mathbf{F}$ from the macroscopic deformation $\mathbf{F}_{h}$ is $\mathbf{F}_{i}=\mathbf{F F}_{h}{ }^{-1}$. We flag $K$ for refinement when the norm of the second invariant of the corresponding Lagrangian strain $\mathbf{E}_{i}=\frac{1}{2}\left(\mathbf{F}_{i}^{T} \mathbf{F}_{i}-\mathbf{I}\right)$ exceeds a pre-specified tolerance. This tolerance is chosen such as to ensure full atomistic resolution well in advance of the passage of dislocations.

The computational cell used for the simulations is a $0.26-\mu \mathrm{m}$ cube of bcc Ta containing $796 a_{0} \times 796 a_{0} \times 796 a_{0}$ unit cells $\left(a_{0}=3.3026 \AA\right)$, or a total of $9.08 \times 10^{8}$ atoms. The cell is oriented along the cubic lattice directions. An equiaxed 10.9-nm void is initially introduced at the center of the cell, corresponding to a porosity of $\sim 3.73 \times 10^{-5}$. The initial triangulation of the cube is tailored to the problem geometry. In particular, full atomistic resolution is introduced in a $30 a_{0} \times 30 a_{0} \times 30 a_{0}$ region around the void from the outset. Away from this region, the triangulation is gradually coarsened. The resulting initial number of nodes, or representative atoms, in the mesh is 
104, 987, which represents a reduction of almost four orders of magnitude in problem size with respect to full atomistics.

\subsection{Analysis of the results}

The computed EOS are given as pressure vs relative volume change (Jacobian). The pressure is calculated as:

$$
p=\frac{1}{3} \sum_{\alpha} \sigma_{\alpha \alpha}
$$

where $\sigma_{\alpha \alpha}$ are the diagonal components of the Cauchy stress tensor. Zhou has shown that, when averaged over (microscopically) large volumes, the Cauchy stress can be obtained directly from the volume-averaged Virial expression [28]:

$$
\begin{aligned}
\sigma_{\alpha \beta} & =\frac{\sum_{K \in \mathcal{T}_{h}} V_{K} \sigma_{\alpha \beta}^{(K)}}{\sum_{K \in \mathcal{T}_{h}} V_{K}} \\
\sigma_{\alpha \beta}^{(K)} & =\frac{1}{2 \Omega_{i}} \sum_{i \in V_{K}}\left(\sum_{j}\left(r_{\alpha}^{(j)}-r_{\alpha}^{(i)}\right) f_{\beta}^{(i j)}\right)
\end{aligned}
$$

where $V_{K}$ is the volume of element $K, \Omega_{i}$ is the atomic volume of bcc Ta, $r_{\alpha}^{(i)}$ is the $\alpha$-th component of the position of atom $i \in V_{K}$, and $f_{\beta}^{(i j)}$ is the $\beta$-th component of the force between atoms $i$ and $j$. The summation in eq. 2a runs over all elements of the system triangulation $\mathcal{T}_{h}$, whereas those in eq. $2 \mathrm{~b}$ encompass, respectively, all atoms in each $V_{K}$ and their neighbors. The Jacobian is simply computed as the determinant of the deformation gradient:

$$
J=\frac{V}{V_{0}}=\operatorname{det} \mathbf{F}=\frac{\sum_{K \in \mathcal{T}_{h}} V_{K}}{\sum_{K \in \mathcal{T}_{h}} V_{K 0}}
$$

where $V_{K 0}$ is the volume of each element in the reference configuration.

In order to reliably identify the defects in the deformed crystal we employ the centrosymmetry deviation parameter (CSD) [29]. For a dislocation core atom in Ta the deviation parameter is between 1.2 and $3.5 \AA^{2}$, between 3.5 and $9.0 \AA^{2}$ for an atom belonging to a twin plate, and 27.0 and $15.4 \AA^{2}$ for, respectively, $\{001\}$ and $\{110\}$ free surfaces. In all subsequent dislocation structure plots, atoms are colored according to the relative magnitude of their centrosymmetry deviation parameter with green/yellow corresponding to free surfaces and red to dislocation cores, while atoms belonging to a twin are identified by means of an orange-to-red color gradation. Once the dislocation lines are identified, the Burgers vectors can be readily determined by performing a Burgers circuit around the dislocation core. Finally, the edge and screw 
components of the dislocation can be resolved by resorting to the following pair of simple expressions:

$$
\begin{aligned}
& \mathbf{b}_{\mathbf{s}}=(\mathbf{b} \cdot \xi) \cdot \xi \\
& \mathbf{b}_{\mathbf{e}}=\xi \times(\mathbf{b} \times \xi)
\end{aligned}
$$

where $\mathbf{b}$ is the total Burgers vector and $\xi$ is a unit vector tangent to the dislocation line.

\section{Results}

Case (i) above involves loading a perfectly-spherical void along one of the principal axes of a cubic sample. These highly-symmetric conditions are not representative of real crystals, although they are a framework to provide an upper estimate of the strength of these voids to uniaxial loading. Case (ii) serves to break this symmetry while maintaining the uniaxial nature of the deformation. It also facilitates the analysis by confining plasticity to one single specific slip system. In the following we provide the results for each case.

\subsection{Uniaxial loading along [001]}

The computed EOS $(p-J)$ for the [001] loading direction is plotted in Fig. 2. Both the loading and the recovery paths are shown in the figure. The loading curve is characterized by an initial (non-linear) elastic stage up to $J \approx 0.922$, followed by yielding all the way up to the maximum compression of $J=$ 0.909. Plastic yielding is a rich process, involving several dislocation emission episodes, and manifests itself as a gradual leveling off of the loading path. The loading curve essentially follows the uniaxial bulk elastic behavior that gives the Young modulus as a function of compression. Interestingly, the difference between the two is minimal despite the presence of dislocations that give rise to irreversible plastic work. Indeed, the material heterogeneity associated with this low porosity becomes diluted by the surrounding elastic matrix and its 'macroscopic' effect is minimal. The first yield point occurs at a critical pressure of $p=12.8 \mathrm{GPa}(J=0.922)$ and is characterized by the emission on the equatorial plane of an edge dislocation ring with Burgers vector $a_{0}[001]$. The formation of this type of loops is not favored elastically in bcc crystals although they have been seen to appear ubiquitously under certain conditions [30]. Figure 3(a) shows the resulting atomistic structure surrounding the void's equator ([001] plane). The state of the QC mesh at this stage is shown in Figure 3(b), where the deformation associated with the loop is clearly visible. This dislocation loop is prismatic and therefore glissile on the glide prism defined 


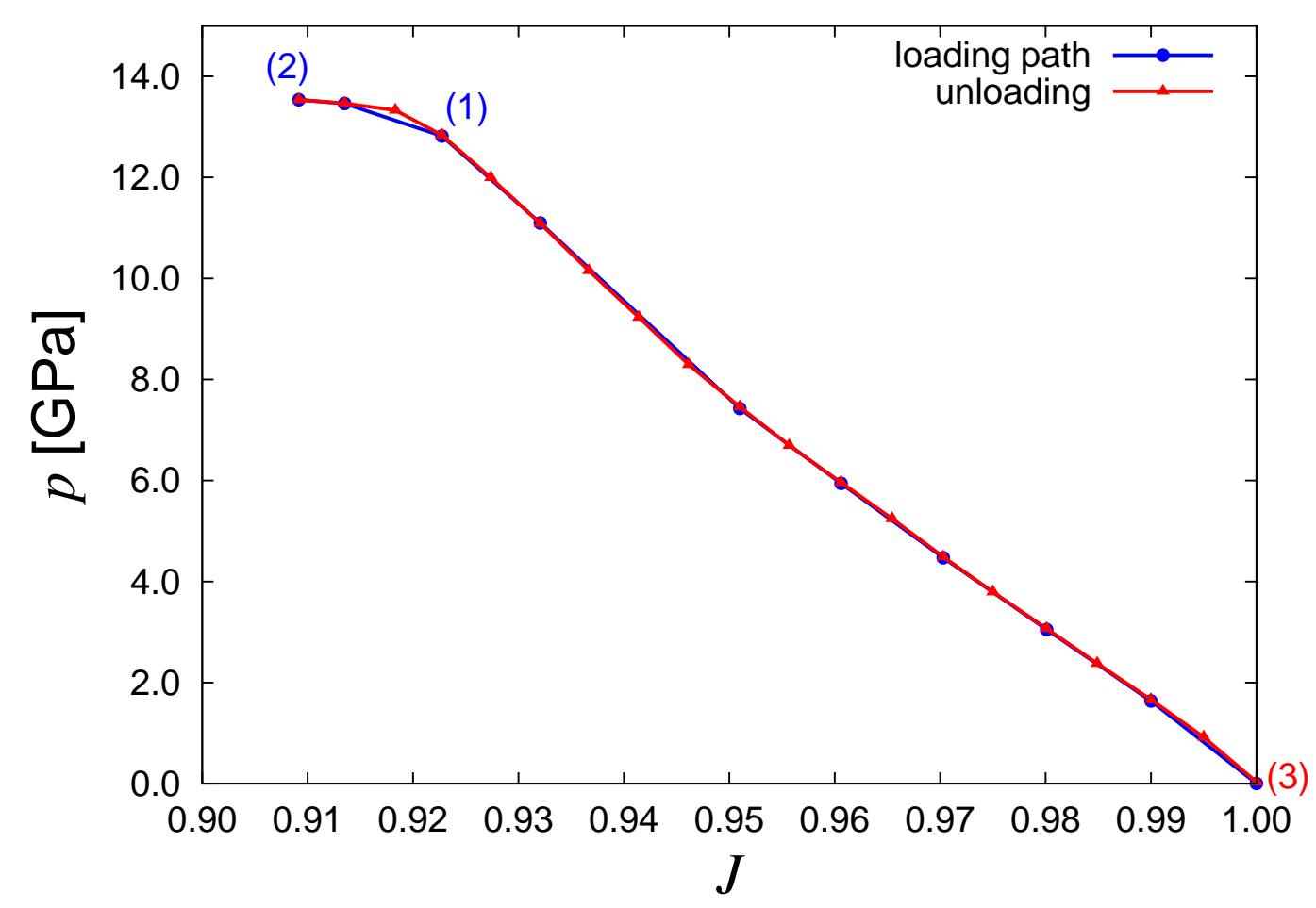

Fig. 2. EOS for [001] loading. Both the loading and reverse loading paths are shown. Both curves virtually lie on top of one another. Points labeled as '(1)', '(2)' and '(3)' mark, respectively, the first yield point, the point of maximum deformation and the final stress-recovered state.

by the loading direction (axis) and (100) and (010) planes (prism sides: note its square shape $)^{1}$. However, this loop does not have any stress acting on it and hence it does not move. In addition, the critical glide stress for $\langle 001\rangle$ type loops is typically quite high and in many instances these loops can be considered immobile for all practical purposes [30].

It is precisely this inability to further relieve the applied stress what triggers the nucleation of dislocation loops with $\frac{1}{2}[11 \overline{1}]$ Burgers vector gliding on (101) planes (their precursors can be appreciated in the lower left corner of Fig. 3(a)). This occurs at $p \approx 13.3 \mathrm{GPa}$ or $J=0.913$ and the resulting atomistic structures are shown in Figure 4(a). While in two of the equatorial quadrants (the upper ones in Figs. 3(a) and 4(a)) there is no additional dislocation activity beyond that seen at the first yield point, in the other ones long dislocation segments are seen to propagate away from the void. These segments are perfect screw in character and display reduced mobility, possibly due to Peierls friction effects (of the order of $\sim 0.8 \mathrm{GPa}[31,32]$ ). However, the leading part of the loops is almost pure edge in character and moves smoothly along its glide prism, pulling and lengthening the screw segments with them.

$\overline{1}$ The glide prism is defined as the locus of the crystallographic planes where $\mathbf{b} \cdot \mathbf{n}=$ 0 . 


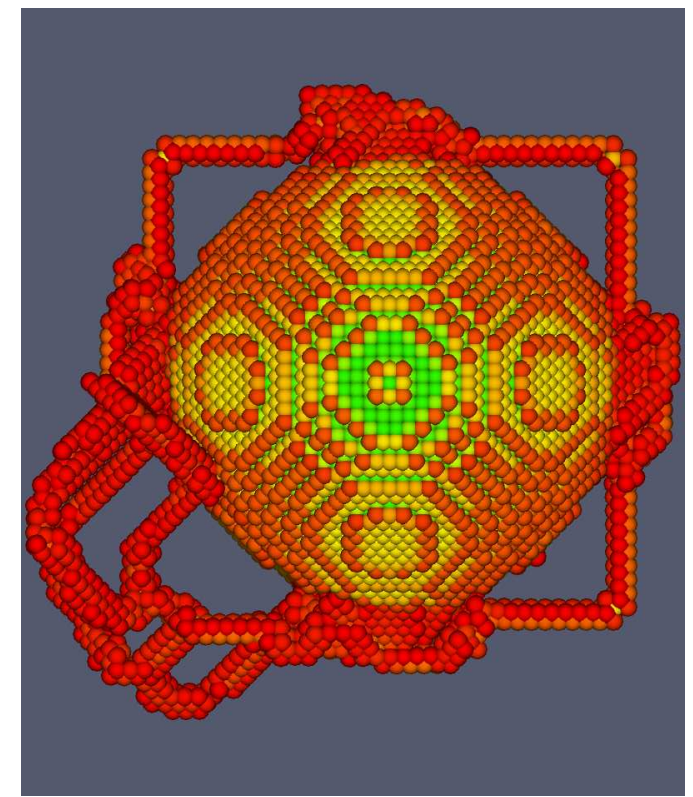

(a) Atomistic representation of the void and its vicinity viewed along the loading direction. The equatorial, $a_{0}\langle 001\rangle$ square loop can can be clearly observed. On the bottom-left part of the image, precursors of $\frac{1}{2}\langle 111\rangle$ loops have already emerged.

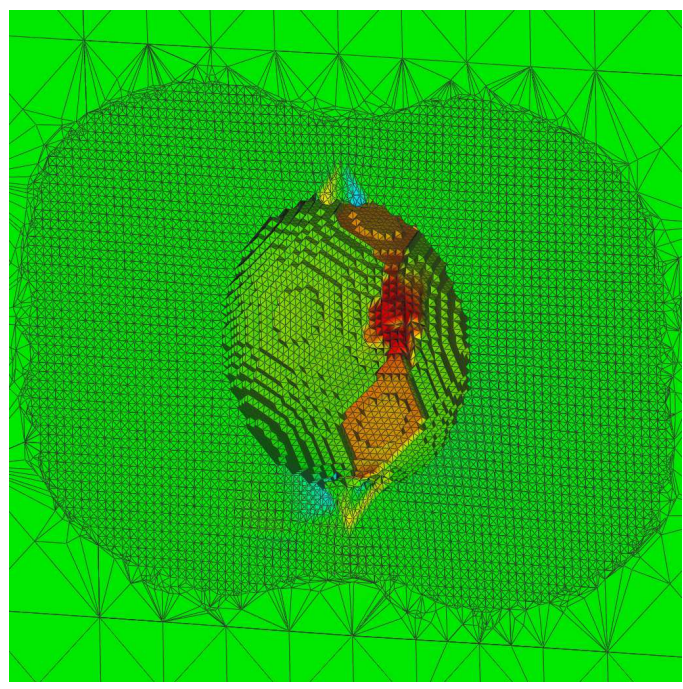

(b) Displacement map superposed on the QC mesh. The horizontal direction coincides with the loading direction.

Fig. 3. View of the void at the the first yield point $(p=12.8 \mathrm{GPa})$, corresponding to the point labeled '(1)' in Fig. 2. 
This results in the effective formation of screw dislocation dipoles closed, on one side, by the void surface and, on the other, by this edge-type dislocation segment.

In principle, with this geometry all slip systems of the $\langle 11 \overline{1}\rangle\{\overline{1} 01\}$ type are equally stressed with Schmidt factor $\frac{1}{\sqrt{3}}$. Therefore, the reason why only two of them are seen to become activated must be related to some symmetrybreaking local perturbation. For example, flipping tetrahedral edges during the remeshing phase of QC (Delaunay triangulation construction) is subject to some numerical variability that may give rise to different results in identical conditions. Despite the numeric, rather than 'natural', character of these fluctuations, these may serve to enrich the statistics of the simulations and lower the values of $p$ required to make the system yield. Regardless, plasticity for this loading orientation is characterized by the emission of leading edge dislocation segments that result in the creation of long screw dislocation dipoles.

The area enclosed between the direct and reverse loading paths represents the plastic work available for thermal heating. For the reasons discussed in Section 2.3, the volume-averaging procedure to compute $p$ prevents the observation of any appreciable hysteresis throughout the complete deformation cycle, resulting in both curves (loading and unloading) lying virtually on top of one another. An alternative way to estimate the degree of irreversibility of this process is by obtaining the number and configuration of dislocations and other plastic structures at the end of the unloading phase.

\subsection{Uniaxial loading along [ $\left[\begin{array}{lll}4 & 8 & 19\end{array}\right]$}

Figure 6 shows the EOS for this loading direction and the associated unloading path. Initially, the EOS increases smoothly following the elastic response as given by the interatomic potential. The system yields at approximately 4.7 GPa, mainly by way of dislocation emission and twinning. Although the EOS suffers a slight leveling thereafter, as in the previous case, these plastic events do not manifest themselves as a sharp yield point drop in Fig. 6. Rather, the EOS behaves very much like in real macroscopic materials, where microscopic plastic activity becomes diluted in the overall materials response. As the figure shows, this loading mode is practically isochoric, indicating that volumetric effects are small compared to shear effects, with volume changes of slightly over $2 \%$ in compression after $11 \mathrm{GPa}$ of applied axial stress.

The most salient plastic feature in this case is the formation of (101)[111] twin plates on the planes of maximum RSS. The mechanism for twinning initiation has been postulated by Lagerlof [33], and shown in detailed atomistic simulations by Marian et al. [34]. In essence, dipoles of twinning partial dislocations 


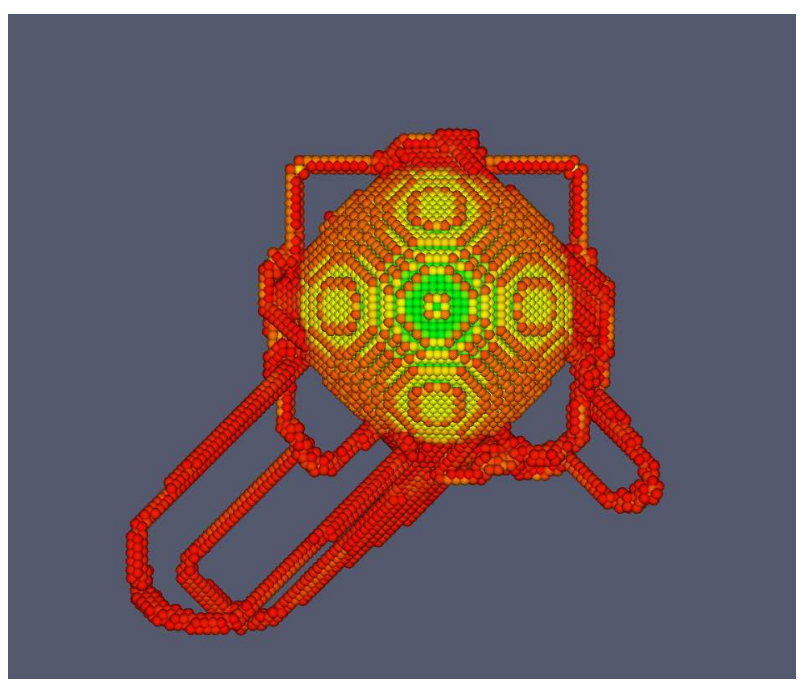

(a) Atomistic representation of the void and its vicinity viewed along the loading direction. Long screw segments can be clearly observed in the lower part of the image, pulled by edge-type segments.

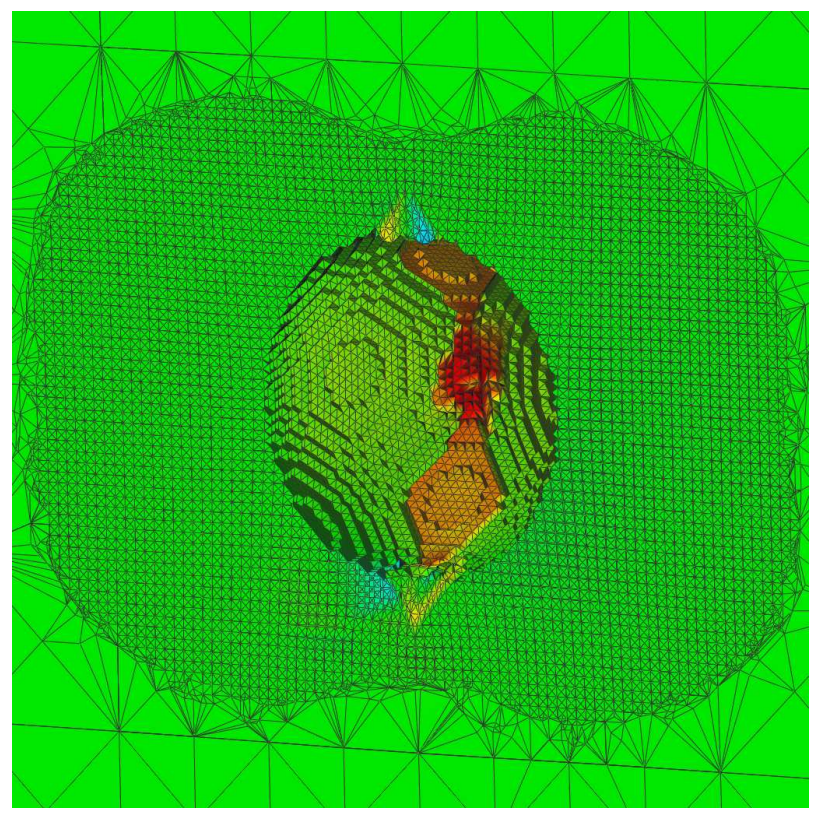

(b) Displacement map superposed on the QC mesh. The horizontal direction coincides with the loading direction.

Fig. 4. View of the void at the point of maximum deformation $(p=13.3 \mathrm{GPa})$, corresponding to the point labeled '(2)' in Fig. 2. 


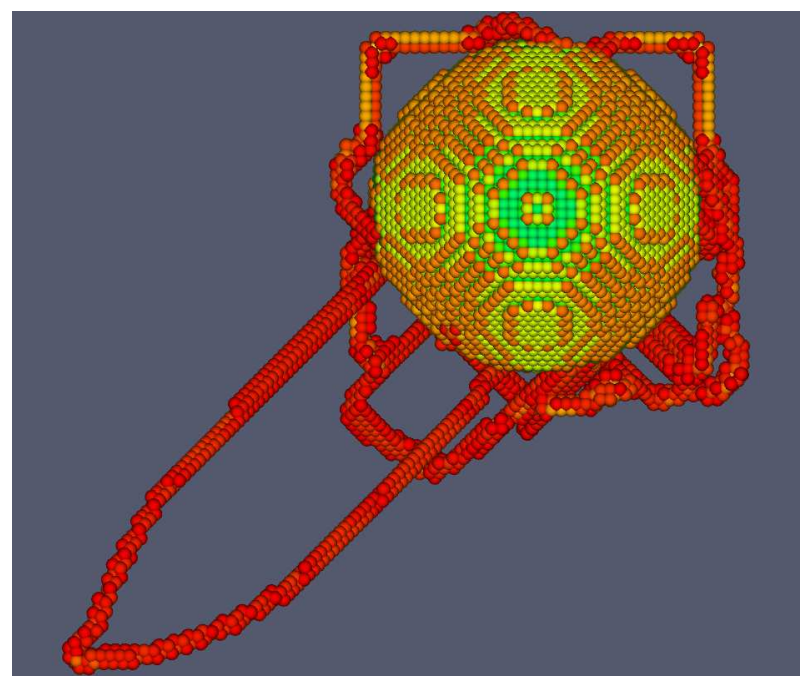

(a) Atomistic representation of the void and its vicinity viewed along the loading direction. Remnant dislocations can be clearly appreciated.

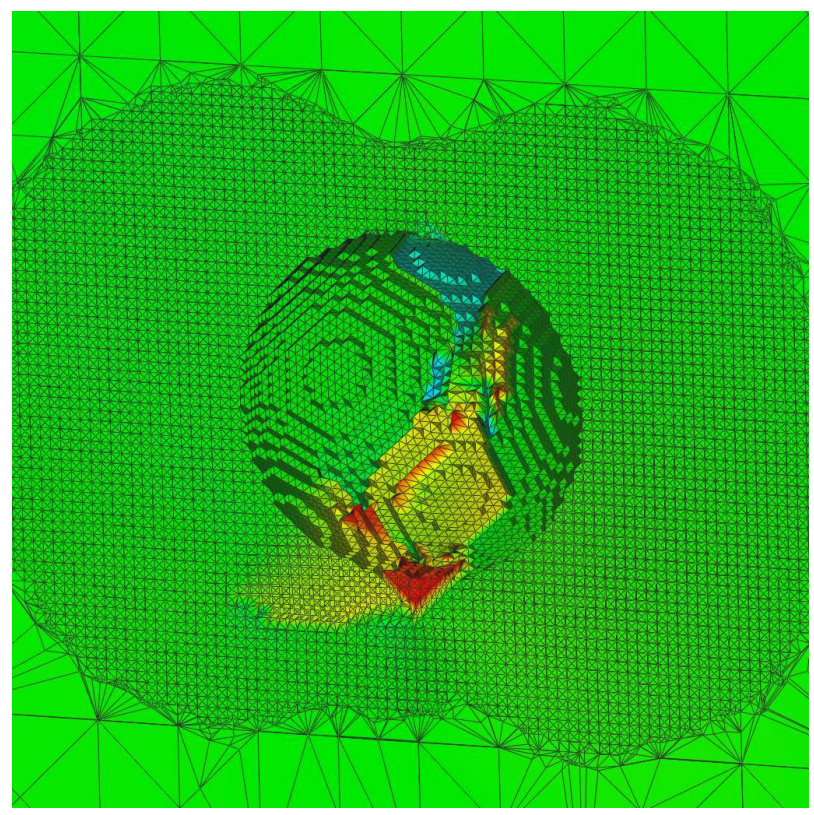

(b) Displacement map superposed on the QC mesh. The horizontal direction coincides with the loading direction.

Fig. 5. View of the void after full unloading, corresponding to the point labeled '(3)' in Fig. 2. 


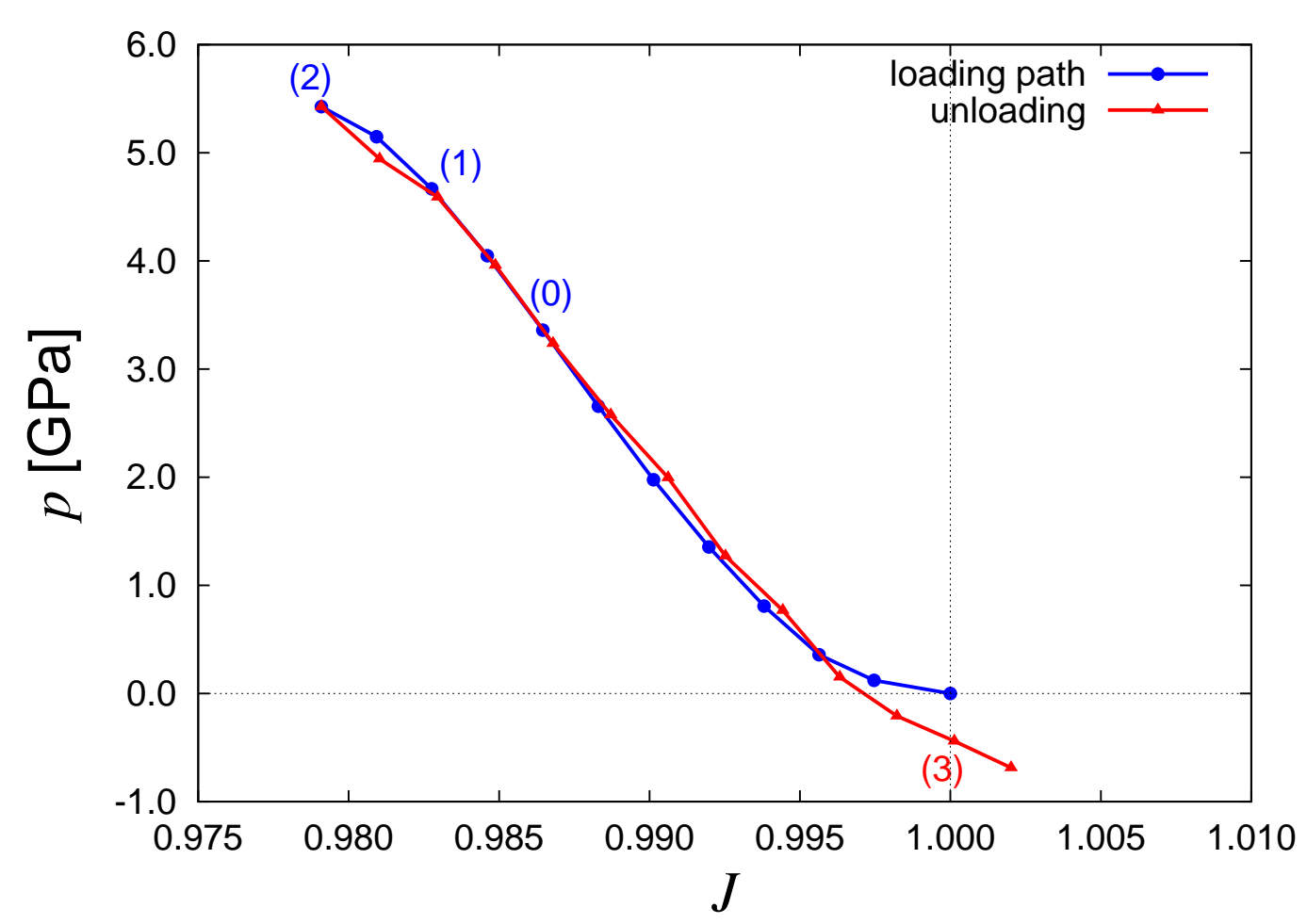

Fig. 6. EOS for $\left[\begin{array}{lll}\overline{4} & 8 & 19\end{array}\right]$ loading. The unloading path is also shown, down to negative (expansive) pressures. Both curves virtually lie on top of one another. Points labeled '(0)', '(1)', '(2)' and '(3)' mark, respectively, a state representative of the elastic regime, the first yield point, the point of maximum deformation and the final deformation-recovered state.

(with $\mathbf{b}=\frac{1}{6}\langle 111\rangle$ ) are nucleated on $\{111\}$ planes at the points of maximum RSS forming $\pm 45^{\circ}$ with the loading direction. These twins are accompanied by some dislocation activity on planes perpendicular to the activated (101). The twin plates keep growing with deformation, both in thickness and in extent, all the way up to the maximum calculated pressure of $p=5.4 \mathrm{GPa}$. In contrast, the dislocation loops lying on perpendicular planes move scarcely. Figures 7(a) and 8(a) show the extent of the QC mesh superposed on the deviatoric displacement field for $p=3.4$ (elastic regime) and 5.4 GPa (maximum deformation) on the loading The figures clearly show the intensity of the deformation and how the QC solution adapts itself to it. In particular, the two twin plates that emanate from the void can be clearly appreciated in Fig. 8(a), as discontinuities in the strain field. The intense displacement band joining both twins is also clearly visible on the inner surface of the void, along an equatorial arc. To better interpret these features we analyze the QC meshes in terms of the corresponding atomic structures using the CSD parameter. At any given moment, QC permits the direct correspondence between the atomistic and mesh representations by recourse to interpolation functions that provide the atomic positions in large elements [25]. Figures 7(b) and 8(b) show, respectively, the onset of twinning at $p=4.7 \mathrm{GPa}$, and the maximum 


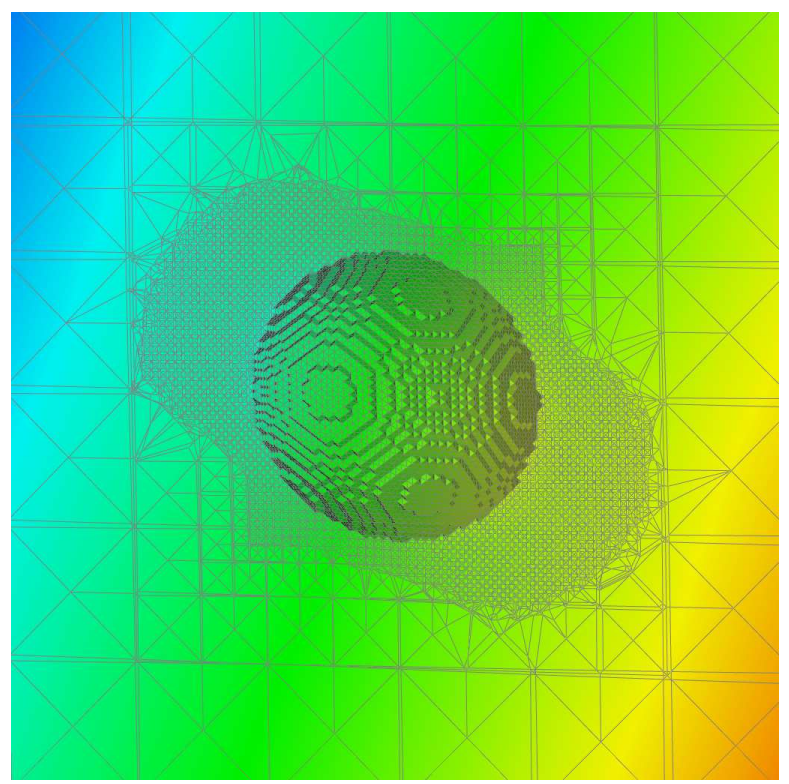

(a)

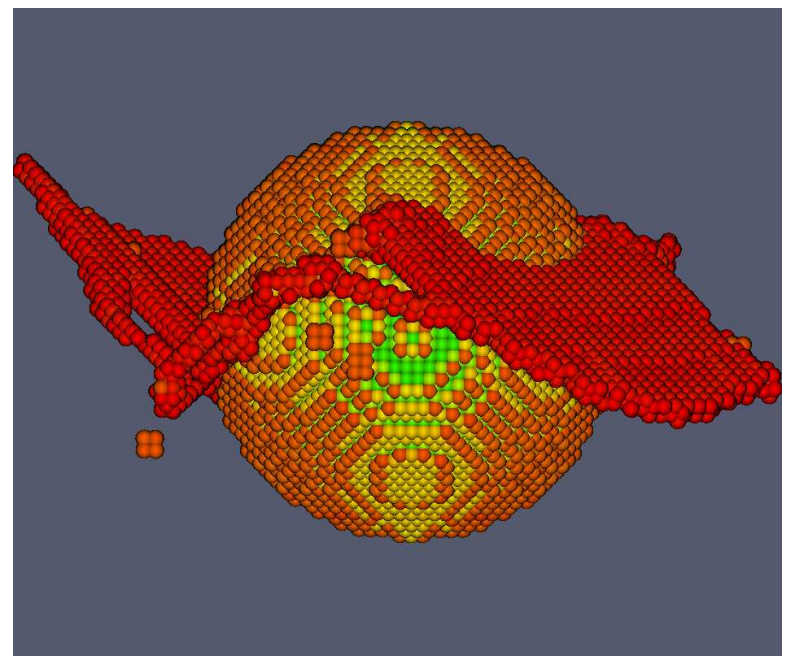

(b)

Fig. 7. (a) Cross section of the void showing the displacement field mapped against the QC mesh for $p=3.4 \mathrm{GPa}$, while the system still behaves elastically. This corresponds to point '(0)' in Fig. 6. (b) Atomistic representation of the void and its vicinity immediately after the onset of twinning, at a pressure of $4.7 \mathrm{GPa}$, corresponding to the point labeled '(1)' in Fig. 6.. The twin plates on (110) planes can be clearly observed together with several dislocation lines on planes orthogonal to them. An isolated vacancy has appeared next to the lower twin plate. 
extent of the twin plates and accompanying dislocation structures at

The stress recovery curve is also shown in Fig. 6, ranging from $J=0.979$ (maximum compression) to $J=1.002$. As the unloading proceeds, the twin plates are gradually reabsorbed into the void and seen to fully disappear at $J=1.000$ deformation. This process is governed mainly by the extent of the twin plates and their distance to the void surface, which in turn determines the magnitude of the image forces. This is clearly shown in Figure 9(a), showing the QC mesh near the end of the recovery phase, where the twin plates have been completely reabsorbed. This behavior is consistent with the loading and unloading curves lying virtually on top of one another down to $J=0.996$, although, again, the volume-averaging procedure employed to compute $p$ masks the relative differences between the loading and reverse loading paths.

However, screw segments belonging to the dislocation lines present after loading are able to irreversible cross-slip and create cross-kinks, resulting in the formation of isolated vacancies (see Ref. [34] for the mechanism). These structures end up on planes that may not directly intersect the void surface and, therefore, survive absorption and remain mostly unaltered. These residual dislocation structures can be seen in Fig. 9(b), where undetached loops with $\frac{1}{2}[11 \overline{1}]$ Burgers vector and several vacancies (identifiable by their eight first nearest neighbors in the bcc lattice) are clearly visible. Notably, this irreversibility is clearly manifested in the $(p-J)$ EOS, where an appreciable deviation from the original elastic behavior can be seen in the $0.996<J<1.000$ range.

\section{Discussion and conclusions}

A principal conclusion of these simulations is that plastic effects due to nanosized heterogeneities in 'macroscopic' systems become completely subdued by the overall elastic behavior of the matrix. In particular, plastic nucleation events and plastic flow do not manifest themselves in the EOS in the form of appreciable yield points or noticeable deviations from the overall elastic response. In this sense, MD simulations may not be sufficient to provide accurate macroscopic constitutive relations despite producing the correct plastic features. QC calculations may, on the other hand, capture correctly these effects by minimizing finite-size effects, although limited to quasi-static conditions. In situations where this limitation is well justified - a case in point being the calculation of cold curves - QC can be reliably used to study the elastic behavior and incipient plasticity.

Another important conclusion is that the available plastic work for irreversible heating cannot be obtained directly from these calculations, at least not in 


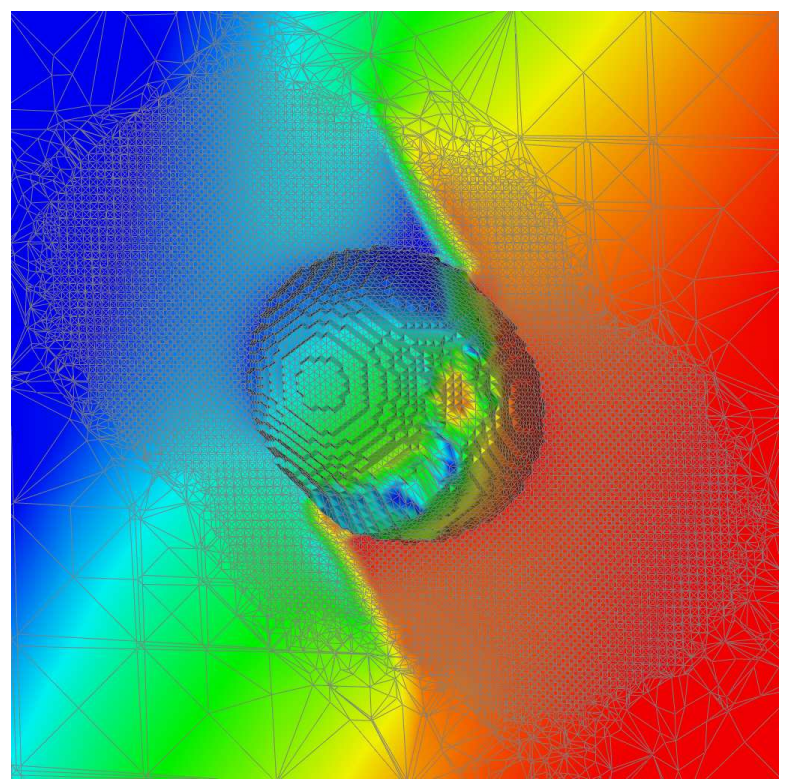

(a) Displacement map superposed on the QC mesh. Two antisymmetric discontinuities, at the top and bottom poles of the void, can be clearly appreciated, signaling the existence of twin plates. Note the extent of the mesh with respect to Fig. 7(a).

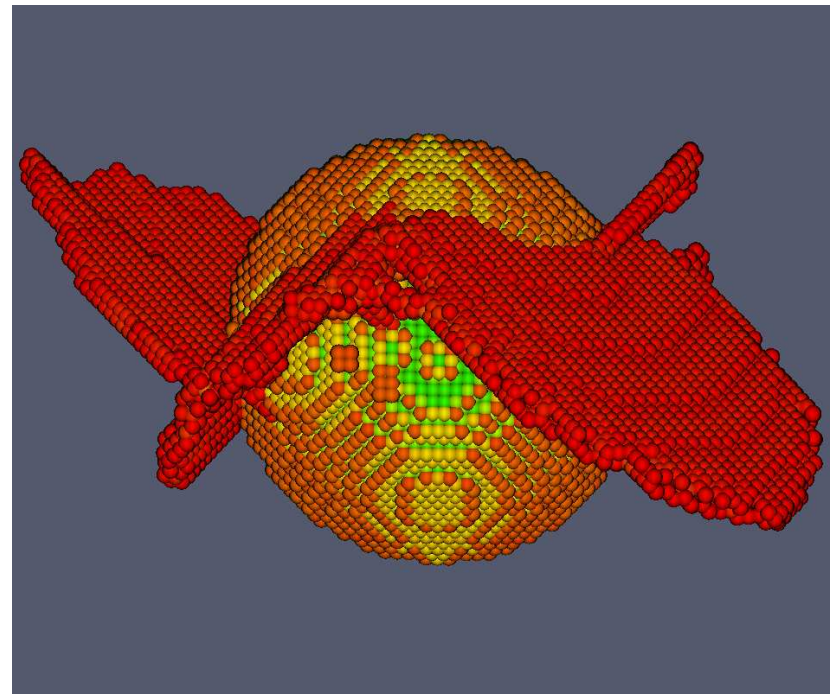

(b) Atomistic representation of the void and its vicinity. The twin plates grow gradually from the continuous nucleation of twinning partials on their surface.

Fig. 8. View of the void immediately at the maximum pressure of $5.4 \mathrm{GPa}$, corresponding to the point labeled '(2)' in Fig. 6. 


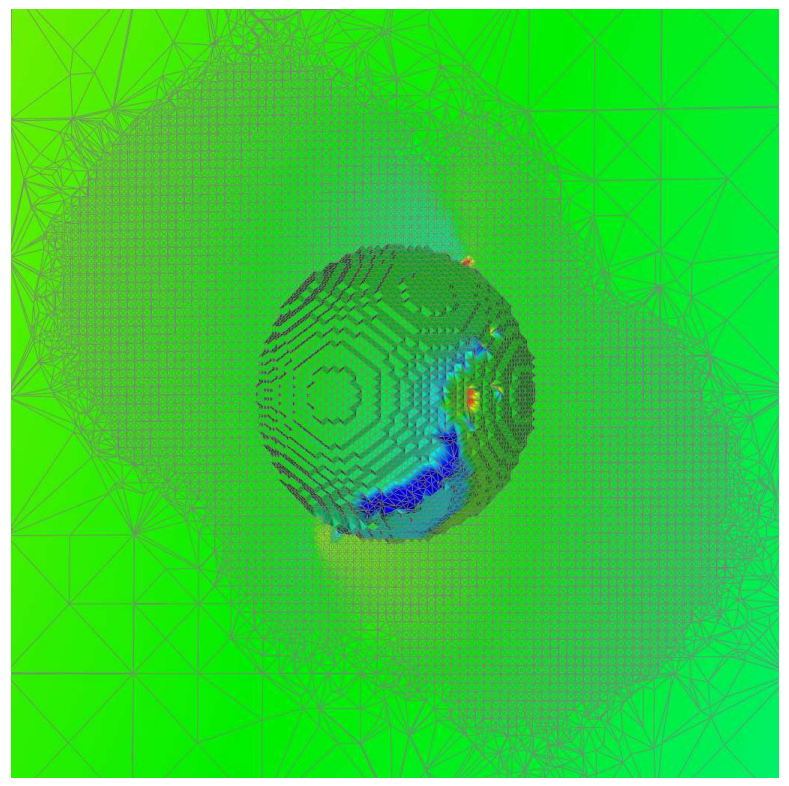

(a) Displacement map superposed on the QC mesh. The twin plates have been completely reabsorbed but the equatorial deformation band still remains.

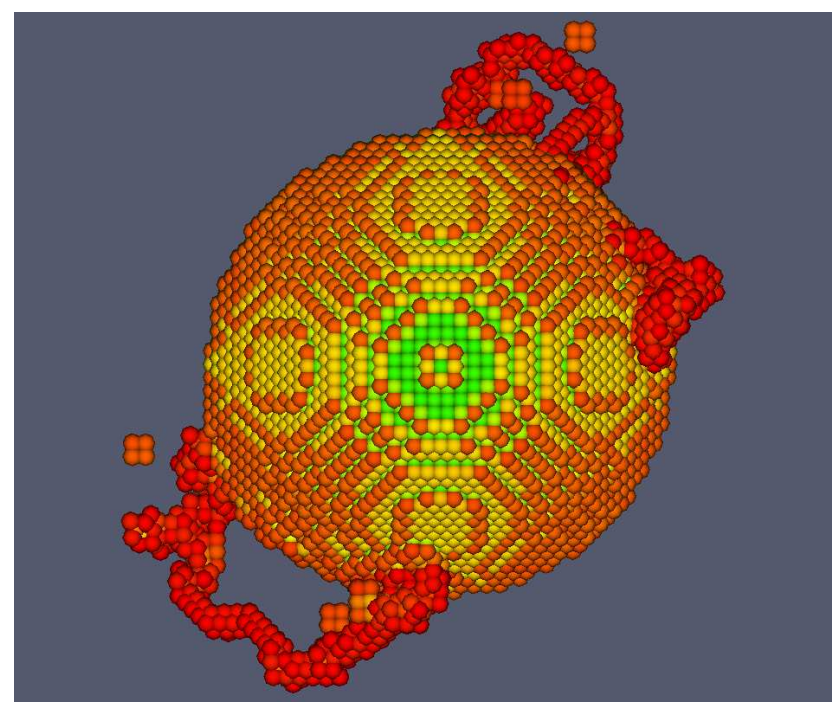

(b) Atomistic representation of the void and its vicinity. The only plastic features remaining are $\frac{1}{2}\langle 11 \overline{1}\rangle$ dislocations, corresponding to the equatorial band appearing in the mesh representation. Several isolated vacancies, the traces of cross-slip events, are also visible

Fig. 9. View of the void during the recovery phase at $p \approx-0.5 \mathrm{GPa}$, corresponding to the point labeled '(3)' in Fig. 6. 
these relatively large systems with such low porosities. Moreover, it has been suggested that the Taylor-Quinney coefficient [35], which dictates the fraction of plastic work that is converted into heat, vanishes under extreme conditions of strain rate and temperature (expected under shock loading) [36]. However, these results can be helpful if thought of as links in a multiscale chain that informs methods with a higher degree of homogenization. For example, the computed EOS can be used as the base constitutive relation in finite-element simulations of shocked porous Ta.

With regard to the methodology employed, we have discussed the advantages and shortcomings of QC in Sections 2 and 3 at length. We have used displacement boundary conditions for our simulations as most experimental tests are performed in that manner. A critical issue is the absence of natural symmetrybreaking perturbations in our calculations, which give rise to relatively large pressures. However, to the extent of our capabilities, we have changed the simulation conditions so as to induce deviations from the ideal behavior a priori considered in Section 3.1. We have seen that the plastic behavior attendant to void collapse under the different conditions explored in Sections 3.1 and 3.2 is radically different, in one case being characterized by screw dislocation dipole formation, while, in the other, by twin plate emission.

As to the interatomic potentials, typically, due to their ease of implementation and relatively low computational overhead, EAM potentials [37] have been used within the QC framework. Although inaccuracies have been reported for EAM calculations of some crystal defects $[38,39]$, this class of potentials has proven sufficiently accurate for a wide range of applications. Recently, QC approaches that use first-principles as the underlying material model have been developed [40]. These methods appear very promising, although for now they are formulated on the basis of orbital-free density functional theory for the sake of computational expeditiousness. An intermediate solution may be using potentials where multi-ion angular forces are taken into account $[41,42]$. This class of potentials has been successful in calculating a wide range of structural, thermodynamic, defect, and mechanical properties at both ambient and extreme conditions. However, they are still about an order of magnitude costlier than standard EAM. Our potential of choice [26] has been fitted to an experimental equation of state for bcc Ta that includes data at 10\% compression. This is a particular useful feature for our calculations and an advantage over other central potentials for Ta found in the literature. In addition, a modification of this potential to more extended ranges has been shown to predict accurate dislocation properties [43].

In terms of validation, there is ample experimental evidence of twinning in shocked Ta [44-48], and under other loading conditions [49,50]. These works demonstrate, first, that twinning is normally initiated at material inhomogeneities, and, second, that twinning is generally accompanied by a significant 
amount of dislocation activity. In addition, there is experimental evidence for the formation of microstructures aligned with the activated slip planes when materials are loaded along directions in the middle of the stereographic triangle [51]. From a computational standpoint, there have been several works highlighting the twinning proclivity of Ta-based materials. For example, Zhou et al. have shown using MD that twins nucleate at interfaces in lamellar TiAl crystals by agglomeration of twinning partials [52]. Additional MD simulations of Ta single crystals suggest a strain-rate dependent transition from dislocation to twinning-governed plasticity [53]. Therefore our results are well within the expected behavior for Ta-based systems under rapid deformation rates.

Future work will be focused on two general issues: (i) populating the stereographic triangle with more loading directions, and (ii) studying the behavior of interacting voids in systems with higher porosities. These two are symmetrybreaking operations which will contribute to enrich the statistical meaningfulness of the calculations. Symmetry can be broken in various other ways, such as having non-spherical voids, mixed loading, thermal fluctuations, etc., which are worth being explored as well.

\section{Acknowledgments}

Useful discussions with R. C. Becker and J. S. Stolken are gratefully acknowledged. This work performed within LDRD project 06-SI-005 under the auspices of the U.S. Department of Energy by Lawrence Livermore National Laboratory under Contract DE-AC52-07NA27344.

\section{Appendix}

\section{A Tensor rotation for uniaxial loading from $\left[\begin{array}{lll}\overline{4} & 8 & 19\end{array}\right]$ to [001]}

We start from the preferred loading direction:

$$
\mathbf{e}_{3} \equiv\left[\begin{array}{lll}
\overline{4} & 8 & 19
\end{array}\right]
$$

For convenience, we choose:

$$
\mathbf{e}_{2} \equiv\left[\begin{array}{lll}
2 & 1 & 0
\end{array}\right]
$$

and,

$$
\mathbf{e}_{1}=\mathbf{e}_{2} \times \mathbf{e}_{3} \equiv\left[\begin{array}{lll}
19 \overline{38} & 4
\end{array}\right]
$$


In the above frame, the second Piola-Kirchhoff stress tensor is:

$$
\mathbf{S}=\left[\begin{array}{ccc}
0 & 0 & 0 \\
0 & 0 & 0 \\
0 & 0 & S_{z}
\end{array}\right]
$$

Now, we want to rotate this tensor to the frame associated with the set of vectors $\mathbf{e}_{1}^{\prime} \equiv[100], \mathbf{e}_{2}^{\prime} \equiv[010], \mathbf{e}_{3}^{\prime} \equiv[001]$. The components of the transformation matrix $\mathbf{Q}$ are:

$$
Q_{i j}=\frac{\mathbf{e}_{i} \mathbf{e}_{j}^{\prime}}{\left\|\mathbf{e}_{i}\right\|\left\|\mathbf{e}_{j}^{\prime}\right\|}
$$

From here, we have that the rotated tensor, $\mathbf{S}^{\prime}$, is:

$$
\mathbf{S}^{\prime}=\mathbf{Q}^{T} \mathbf{S Q}
$$

For QC, we need to obtain the deformation gradient $\mathbf{F}^{\prime}$ associated with $\mathbf{S}^{\prime}$. For first-order hyper-elasticity [?], we have

$$
\mathbf{S}=\mathbb{C}: \mathbf{E}
$$

where $\mathbb{C}$ denotes the fourth-rank elasticity tensor and $\mathbf{E}=\frac{1}{2}\left(\mathbf{F}^{T} \mathbf{F}-\mathbf{I}\right)$ is the Green-Lagrange strain tensor. For simple crystals, relationship (A.4) can be simplified by recourse to the Voigt rule

$$
\left[\begin{array}{c}
S_{11} \\
S_{22} \\
S_{33} \\
S_{23} \\
S_{13} \\
S_{12}
\end{array}\right]=\left[\begin{array}{cccccc}
C_{11} & C_{12} & C_{12} & 0 & 0 & 0 \\
C_{12} & C_{11} & C_{12} & 0 & 0 & 0 \\
C_{12} & C_{12} & C_{11} & 0 & 0 & 0 \\
0 & 0 & 0 & C_{44} & 0 & 0 \\
0 & 0 & 0 & 0 & C_{44} & 0 \\
0 & 0 & 0 & 0 & 0 & C_{44}
\end{array}\right]\left[\begin{array}{c}
E_{11} \\
E_{22} \\
E_{33} \\
2 E_{23} \\
2 E_{13} \\
2 E_{12}
\end{array}\right]
$$

The elastic constants correspond to the Ta EAM potential [26] and are equal to $C_{11}=248.5 \mathrm{MPa}, C_{12}=144.6 \mathrm{MPa}$, and $C_{44}=86.5 \mathrm{MPa}$. Starting with $\mathbf{S}^{\prime}$ and inverting the relation (A.5) we obtain the associated $E_{i j}^{\prime}$. Subsequently, assuming $\mathbf{F}^{\prime}=\mathbf{F}^{\prime T}$, i.e. no rigid rotations, $\mathbf{F}^{\prime}$ can be computed directly from $\mathbf{E}^{\prime}$. The calculation yields let's check the calculation

$$
\mathbf{F}^{\prime}=\left[\begin{array}{ccc}
\left(1-0.0022 S_{z}\right) & -0.0008 S_{z} & -0.0020 S_{z} \\
-0.0008 S_{z} & \left(1-0.0012 S_{z}\right) & -0.0040 S_{z} \\
-0.0020 S_{z} & -0.0040 S_{z} & \left(1-0.0053 S_{z}\right)
\end{array}\right]
$$


The condition for compression is $J=\operatorname{det} \mathbf{F}^{\prime}<1$ :

$$
\operatorname{det} \mathbf{F}^{\prime}=1-0.0087 S_{z}+2.0012 \times 10^{-8} S_{z}^{2}+1.6601 \times 10^{-8} S_{z}^{3}<1
$$

which amounts to solving the following quadratic inequality:

$$
-0.0087+2.0012 \times 10^{-8} S_{z}+1.6601 \times 10^{-8} S_{z}^{2}<0
$$

whose two roots are -724.5 and $723.3 \mathrm{GPa}$. In other words, the two $S_{z}$ ranges where compression is guaranteed are:

$$
0<J<1\left\{\begin{array}{l}
S_{z}<-724.5 \mathrm{GPa} \\
0<S_{z}<723.3 \mathrm{GPa}
\end{array}\right.
$$

\section{References}

[1] J. R. Asay and M. D. Knudson, "Use of Pulsed Magnetic Fields for QuasiIsentropic Compression Experiments" (Chapter 10), in: High-Pressure Shock Compression of Solids VIII - 2005 (eds. L. C. Chhabildas, L. Davidson, Y. Horie) Springer

[2] L. Burakovsky and D. L. Preston, J. Phys. Chem. Solids 9-9 (2004) 1581

[3] S. N. Luo and D. C. Swift, Physica B-Condensed Matter 388 (2007) 139

[4] Computational Materials Science: From Basic Principles to Material Properties, in "Lecture Notes in Physics", Wolfram Hergert, Arthur Ernst, Markus Däne (Eds.), Vol. 642 (Springer, 2004)

[5] K. Ohno, K. Esfarjani, Y. Kawazo, Computational Materials Science: From AB Initio to Monte Carlo Methods, in "Solid-State Sciences", Vol. 129 (Springer, 1999)

[6] R. M. German, "Powder Metallurgy Science", $2^{\text {nd }}$ edition (Metal Powder Industries Federation, Princeton, NJ, 1994)

[7] G. S. Upadhyaya, "Powder Metallurgy Technology" (Cambridge International Science Publishing, Cambridge, 2002)

[8] G. S. Was, "Fundamentals of Radiation Materials Science" (Springer-Verlag, Berlin, 2007)

[9] T. W. Clyne, "Metallic Composite Materials", Chapter 30 in Physical Metallurgy, $4^{\text {th }}$ edition, edited by R. W. Cahn and P. Haasen (Elsevier Science, Amsterdam, 1996)

[10] B. L. Holian and P. S. Lomdahl, Science 280 (1998) 2085 
[11] V. V. Zhakhovskii, S. V. Zybin, K. Nishihara and S. I. Anisimov, Phys. Rev. Lett. 83 (1999) 1175

[12] E. M. Bringa, J. U. Cazamias, P. Erhart, J. Stölken, N. Tanushev, B. D. Wirth, R. E. Rudd and M. J. Caturla, J. Appl. Phys. 96 (2004) 3793

[13] J. Knap and M. Ortiz, Phys. Rev. Lett. 90 (2001) 226102

[14] J. Marian, J. Knap and M. Ortiz, Phys. Rev. Lett. 93 (2004) 165503

[15] J. Marian and J. Knap, Inter. J. Multiscale Comp. Eng. 5 (2007), in press.

[16] J. Belak, J. Comp.-Aided Mat. Des. 5 (1998) 193

[17] B. P. Somerday, P. D. Patillo, M. F. Horstemeyer and M. I. Baskes, in "Multiscale Phenomena in Materials-Experiments and Modeling", D. H. Lassila, I. M. Robertson, R. Phillips, B. Devincre, eds., Symp. P, 1999 Mat. Res. Soc. Fall Meeting, vol. 578, p. 333

[18] E. T. Seppala, J. Belak and R. E. Rudd, Phys. Rev. B 69 (2004) 134101

[19] X. L. Deng, W. J. Zhu, H. L. He, D. X. Wu and F. Q. Jing, Acta Phys. Sinica 55 (2006) 4767

[20] T. Hatano, Phys. Rev. Lett. 93 (2004) 085501

[21] P. Erhart, E. Bringa, M. Kumar and K. Albe, Phys. Rev. B 72 (2005) 052104

[22] L. P. Davila, P. Erhart, E. M. Bringa, M. A. Meyers, V. A. Lubarda, M. S. Schneider, R. Becker and M. Kumar, Appl. Phys. Lett. 86 (2005) 181902

[23] E. B. Tadmor, M. Ortiz and R. Phillips, Phil. Mag. A 73 (1996) 1529

[24] E. B. Tadmor, R. Phillips and M. Ortiz, Langmuir 12 (1996) 4529

[25] J. Knap and M. Ortiz, J. Mech. Phys. Solids 49 (2001) 1899

[26] Y. Li, D. J. Siegel, J. B. Adams and X-Y. Liu, Phys. Rev. B 67 (2003) 125101

[27] W. H. Press, W. T. Vetterling, S. A. Teutoslky and B. P. Flannery, Numerical Recipes in $\mathrm{C}++, 2^{\text {nd }}$ edition (Cambridge University Press, Cambridge, UK, 2002)

[28] M. Zhou, Proc. Royal Soc. Lon. A 459 (2003) 2347

[29] C. Kelchner, S. Plimpton and J. Hamilton, Phys. Rev. B 58 (1998) 11085

[30] J. Marian, B. D. Wirth, R. Schäublin, J. M. Perlado and T. Díaz de la Rubia, J. Nucl. Mater. 307-311 (2002) 871

[31] J. A. Moriarty, J. Belak, R. E. Rudd, P. Söderlind, F. Streitz and L. H. Yang, J. Phys.: Condens. Matter 14 (2002) 2825

[32] G. Wang, A. Strachan, T. Cagin and W. A. Goddard, Modelling Simul. Mater. Sci. Eng. 12 (2004) S371 
[33] K. P. D. Lagerlof, Acta Metall. Mater. 41 (1993) 2143

[34] J. Marian, W. Cai and V. V. Bulatov, Nature Materials 3 (2004) 158

[35] G. I. Taylor and H. Quinney, Phil. Trans. Roy. Soc. A 230 (1931) 323

[36] S. Yadav, E. A. Repetto, G. Ravichandran and M. Ortiz, Inter. J. Impact Eng. 25 (2001) 787

[37] M. S. Daw, S. M. Foiles, M. I. Baskes, MAter. Sci. Reports 9 (1993) 251

[38] G. H. Campbell, J. Belak and J. A. Moriarty, Scripta Mater. 43 (2000)659

[39] G. H. Campbell, M. Kumar, W. E. King, J. Belak, J. A. Moriarty and S. M. Foiles, Phil. Mag. A 82 (2002)1573

[40] V. Gavini, K. Bhattacharya and M. Ortiz, J. Mech. Phys. Solids 55 (2007) 697

[41] J. A. Moriarty, L. X. Benedict, J. N. Glosli, R. Q. Hood, D. A. Orlikowski, M. V. Patel, P. Soderlind, F. H. Streitz, M. J. Tang and L. H. Yang, J. Mater. Res. 21 (2006) 563

[42] Y. Mishin, A. Y. Lozovoi, Acta Mater. 54 (2006) 5013

[43] P.-M. Anglade, G. Jomard, G. Robert and G. Zerah, J. Phys.: Condens. Matter 17 (2005) 2003

[44] R. W. Anderson and S. E. Bronisz, Acta Metall. 7 (1959) 645

[45] L. E. Murr, M. A. Meyers, C.-S. Niou, Y. J. Chen, S. Pappu and C. Kennedy, Acta Mater. 45 (1997) 157

[46] S. Pappu and L. E. Murr, Mater. Sci. \& Eng. A 284 (2000) 148

[47] L. M. Hsiung and D. H. Lassila, Acta Mater. 48 (2000) 4851

[48] L. L. Hsiung, Proceedings of the Conference of the American Physical Society Topical Group on Shock Compression of Condensed Matter, July 28, 2006, vol. 845 , pp. $228-231$

[49] T. E. Mitchell and W. A. Spitzig, Acta Metall. 13 (2001) 1169

[50] Y. M. Wang, A. M. Hodge, J. Biener, A. V. Hamza, D. E. Barnes, K. Liu, T. G. Nieh, Appl. Phys. Lett. 86 (2005) 101915

[51] J. A. Wert, X. Huang, G. Winther, W. Pantleon and H. F. Poulsen, Materials Today 10(9) (2007) 24

[52] L. G. Zhou, L. M. Hsiung and H. C. Huang, CMES 6 (2004) 245

[53] R. E. Rudd, private communication (2007) 